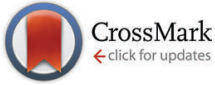

Cite this: Phys. Chem. Chem. Phys., $2016,18,31202$

Received 17th August 2016,

Accepted 21st October 2016

DOI: $10.1039 / c 6 c p 05696 b$

www.rsc.org/pccp

\section{Mechanically induced cis-to-trans isomerization of carbon-carbon double bonds using atomic force microscopy $\dagger$}

\author{
Milad Radiom, *a Phally Kong, ${ }^{\text {b }}$ Plinio Maroni, ${ }^{a}$ Mark Schäfer, ${ }^{\text {b }}$ \\ Andreas F. M. Kilbinger ${ }^{b}$ and Michal Borkovec ${ }^{a}$
}

Cis-to-trans isomerization of carbon-carbon double bonds can be induced by the application of mechanical force. Using single molecule force spectroscopy by means of atomic force microscopy (AFM) we pulled polymer molecules which contained cis double bonds in the backbone. In the force versus extension profiles of these polymers, a sudden extension increase is observed which is due to the conversion of shorter cis isomers into longer trans isomers. The added length to the polymer results in relaxation in probed force. We find that the isomerization occurs at forces of $800 \pm 60 \mathrm{pN}$, independent of AFM tip and solid substrate chemistries. Investigation of similar polymers which exclusively contained single bonds in the backbone showed no evidence of a similar transition.

\section{Introduction}

Isomerization of carbon-carbon double bonds results in molecules with cis, trans, or combination of the two isomeric states which show distinct physical and chemical properties. ${ }^{1}$ Isomerization normally occurs via irradiation, for example isomerization of 11-cis-retinal to trans-retinal in the human eye, ${ }^{2}$ and isomerization of stilbene and its derivatives, ${ }^{3}$ by heat and at low pressure in cis- and trans-2-butene, ${ }^{4}$ by enzymes in the reverse isomerization of trans-retinal to 11-cis-retinal, by catalysts using silver and ruthenium, ${ }^{5,6}$ or via surface-assisted pathways. ${ }^{7}$ So far the effect of mechanical force on isomerization of carbon-carbon double bonds has not been investigated.

In this paper, we show that the isomerization of carboncarbon double bonds, from the cis isomeric state to the trans state, can be induced by the application of mechanical force. Our approach follows the previous mechanochemical experiments which have led to remarkable insights into the conversion of mechanical force to chemical transformation at the level of single polymer molecules and sometimes single bonds. Examples include $\pi-\pi$ interaction, ${ }^{8}$ hydrogen and covalent bonding, ${ }^{9-11}$ and conformational transition. ${ }^{12-14}$ To realize these experiments, a well-defined force-sensitive chemical moiety, a mechanophore, is embedded in a polymer backbone. This addition results in a force-responsive or a mechanically active polymer. Upon pulling

\footnotetext{
${ }^{a}$ Department of Inorganic and Analytical Chemistry, University of Geneva, Geneva, Switzerland. E-mail: milad.radiom@unige.ch

${ }^{b}$ Department of Chemistry, University of Fribourg, Fribourg, Switzerland

$\dagger$ Electronic supplementary information (ESI) available. See DOI: 10.1039/c6cp05696b
}

the polymer using AFM, the restoring force due to polymer backbone rigidity is initially probed in the force versus extension profile. The mechanical force is transferred to the mechanophore through the polymer backbone which subsequently reduces the activation energy barrier of the chemical transition in the mechanophore. When the activation energy barrier is lowered enough so that the transition can proceed thermally, a chemical reaction occurs within the mechanophore which manifests itself as an irregular change in the force versus extension profile of the polymer. The restoring force due to the backbone rigidity of the transformed polymer can be probed by further extending the polymer.

Both plateaus and sudden relaxations have been observed in the force versus extension profiles of force-sensitive polymers upon transition of the mechanophore. Examples of measurements that resulted in plateaus include ring-opening of benzocyclobutene, gem-difluorocyclopropane and gem-dichlorocyclopropane, ${ }^{9}$ and conformational transitions in polysaccharides. ${ }^{14}$ Examples of measurements that resulted in sudden relaxations include triazole ring-opening, ${ }^{10}$ isomerization of prolyl, ${ }^{12,13}$ opening of modular domains in titin and tenascin, ${ }^{15,16}$ unfolding of polymeric nanoparticles, ${ }^{17}$ and unfolding of RNA molecules ${ }^{18}$ and DNA hairpin. ${ }^{19}$ We find that the mechanically induced isomerization of cis double bonds in a polymer backbone resembles the response of a mechanophore. More specifically, in the force versus extension profiles of polymers with cis double bonds in the backbone, a sudden extension increase and relaxation in probed force are observed. The polymer extends upon isomerization since the trans isomer is longer than the cis isomer.

The structures of polymers with cis double bonds in the backbone are summarized in Fig. 1: P1 (with 50\% of double 


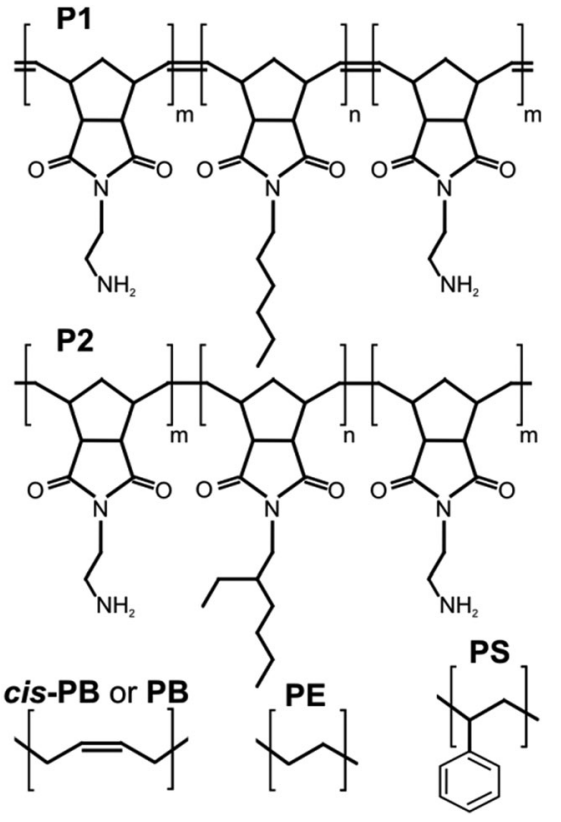

Fig. 1 Structural formulas of the polymers used in this study. The two synthesized polymers are abbreviated as $\mathbf{P} \mathbf{1}$ and $\mathbf{P} \mathbf{2}$. Other polymers include cis-1,4-polybutadiene (cis-PB), cis/trans-1,4-polybutadiene (PB), polyethylene (PE) and polystyrene (PS).

bonds in the cis isomeric state), cis/trans-1,4-polybutadiene (PB, $36 \%$ ) and cis-1,4-polybutadiene (cis-PB, 98\%). The choice of P1 for the mechano-isomerization experiments is due to its specific structure. During isomerization, the bulky side chains of this polymer will rotate around the backbone. This property can be possibly employed to produce new material functions. The choice of cis-PB and PB is to show that the mechanoisomerization is not specific to $\mathbf{P 1}$, but can be induced in other polymers with cis double bonds in the backbone.

As control, three structurally similar polymers without double bonds in the backbone were investigated (Fig. 1): P2, polyethylene (PE) and polystyrene (PS). The characteristic extension increase and force relaxation as were observed in the force versus extension profiles of the polymers with cis double bonds in the backbone were not observed for the polymers without double bonds in the backbone.

\section{Materials and methods}

\section{Materials}

Dimethyl sulfoxide (DMSO) was purchased from Acros Organics. 1,2-Dichlorobenzene (DCB), toluene and methyl benzoate were purchased from Sigma Aldrich. Absolute ethanol was purchased from VWR International. All solvents were dry $(\geq 99 \%)$ and used as received. (3-Glycidoxypropyl)dimethylethoxysilane and 1-nonanethiol were purchased from Sigma Aldrich. PB, cis-PB, PE and PS (molecular weights $M_{\mathrm{w}}=200,250,4500$, and $280 \mathrm{~kg} \mathrm{~mol}^{-1}$, respectively) were purchased from Sigma Aldrich.

Experiments were carried out on silica, functionalized silica, gold and functionalized gold. Naturally oxidized silicon wafers were obtained from Silchem (Germany). A small piece of silicon wafer was cleaned for 20 min with piranha solution, which consisted of a mixture of $\mathrm{H}_{2} \mathrm{SO}_{4} 98 \%$ and $\mathrm{H}_{2} \mathrm{O}_{2} \quad 30 \%$ in a volumetric ratio of $3: 1$. The piece was then rinsed with Milli-Q water and dried in a stream of nitrogen. The clean substrate was then immediately used for polymer deposition or surface functionalization. Epoxy-functionalized silica (EF-silica) was prepared as follows. The clean silica substrate was placed in an evacuated container aside a $150 \mu \mathrm{L}$ drop of (3-glycidoxypropyl)dimethylethoxysilane for $1 \mathrm{~h}$. The EF-silica substrate was then immediately used for polymer deposition. Gold-coated glass slides were obtained from Phasis (Switzerland). A small piece of gold-coated glass slide was immersed in $2 \%(\mathrm{w} / \mathrm{w})$ sodium dodecyl sulfate for $30 \mathrm{~min}$, then rinsed with Milli-Q water and ethanol and finally dried in a stream of nitrogen. It was then treated in a UV-ozone cleaner (PSD Pro, Novascan, Ames, USA) for $20 \mathrm{~min}$ in an oxygen-enriched atmosphere. This cleaning procedure was repeated three times. The cleaned gold substrate was then immediately used for polymer deposition or surface functionalization. Methyl-functionalized gold (MF-gold) was prepared as follows. 1-Nonanethiol diluted to a concentration of $0.5 \mathrm{mM}$ in absolute ethanol was adsorbed on gold by an overnight $(>12 \mathrm{~h}$ ) immersion. The film was then rinsed with abundant ethanol. The MF-gold substrate was then immediately used for polymer deposition.

\section{Synthesis and characterization}

ABA-triblock copolymer 3 was synthesized via ring opening metathesis polymerization (ROMP) using a Grubbs 3rd generation initiator by sequential addition of monomers $2, \mathbf{1}$ and 2 (see the ESI $\dagger$ for details of the syntheses of polymers P1 and P2). Polymer 3 contained $50 \%$ cis double bonds in the backbone. Cleavage of the tert-butyloxycarbonyl (BOC) protective group under acidic conditions ( $\mathrm{HCl}$ ) yielded the triblock copolymer P1 carrying amine hydrochloride salts in the outer blocks of the ABAtriblock structure. Gel permeation chromatography (GPC) analysis in chloroform against PS standards gave a number average molecular weight of $M_{\mathrm{n}}=145 \mathrm{~kg} \mathrm{~mol}^{-1}$ and a dispersity of $D=1.6$.

A sample of the BOC-protected polymer 3 was hydrogenated using the Grubbs 1st generation catalyst under a hydrogen atmosphere (40 bar) at $40{ }^{\circ} \mathrm{C}$ for $48 \mathrm{~h} .{ }^{1} \mathrm{H}$-NMR spectroscopy confirmed the complete disappearance of olefinic signals. However, all attempts to cleave the BOC protective groups under acidic conditions yielded an insoluble material. In order to increase solubility, a second ABA-triblock copolymer 5 was synthesized via sequential addition of monomers $2, \mathbf{4}$ and 2 . Monomer 4 which carries a branched side chain typically shows better organo-solubility than monomers carrying the $n$-hexyl side chain. To further improve organo-solubility, a lower molecular weight $\left(85 \mathrm{~kg} \mathrm{~mol}^{-1}\right)$ was targeted for the triblock polymer 5 . Hydrogenation of 5 using the Grubbs 1st generation catalyst under a hydrogen atmosphere ( $40 \mathrm{bar}$ ) at $40{ }^{\circ} \mathrm{C}$ for $48 \mathrm{~h}$ yielded saturated polymer 6. Cleavage of the tert-butyloxycarbonyl (BOC) protective group under acidic conditions (trifluoroacetic acid, TFA) yielded the saturated triblock copolymer P2 carrying the TFA salts of the primary amine in the outer blocks of the ABA-triblock structure. 
GPC analysis in chloroform against PS standards gave $M_{\mathrm{n}}=$ $76 \mathrm{~kg} \mathrm{~mol}^{-1}$ and $D=1.2$.

$\mathbf{P 1}$ was dissolved in DMSO and P2 in DCB at a concentration of $100 \mathrm{mg} \mathrm{L}^{-1}$. PB, cis-PB, PE and PS were dissolved in toluene at a concentration of $100 \mathrm{mg} \mathrm{L}^{-1}$.

\section{AFM experiments}

AFM pulling experiments were performed using a Cypher AFM (Asylum Research, Santa Barbara, CA). The force versus extension profiles were acquired with cantilevers with silicon tips (BL-AC40TS, Olympus, Japan), functionalized silicon tips, and gold coated tips (TR400PB, Olympus, Japan). The AFM tips were cleaned in a UV-ozone cleaner as described above. The spring constants were in the range of $0.05-0.10 \mathrm{~N} \mathrm{~m}^{-1}$ for the silicon and functionalized silicon tips and $0.16-0.17 \mathrm{~N} \mathrm{~m}^{-1}$ for the goal coated tips as obtained from the thermal fluctuation method. Functionalization of silicon tips with epoxide groups (EF-tip) was performed in an overnight reaction $(>12 \mathrm{~h})$, where the tip was placed adjacent to a $150 \mu \mathrm{L}$ drop of (3-glycidoxypropyl)dimethylethoxysilane as described above. Prior to pulling using the AFM, $10 \mu \mathrm{L}$ of a polymer solution was deposited on the substrate. For P1 and P2, the deposition period was $120 \mathrm{~min}$, while for the other polymers the deposition period was until the solution completely dried in air $(20 \mathrm{~min})$. The substrate was then rinsed with the respective solvent and dried under a stream of nitrogen. The polymer-coated substrate was mounted in the AFM cell and $40 \mu \mathrm{L}$ of an appropriate working solution was added. For $\mathbf{P 1}$ and $\mathbf{P 2}$, the working solutions were DMSO and DCB, respectively, while for the rest of the polymers the working solution was methyl benzoate. The pulling experiments were carried out with repeated approach-retraction cycles with a sampling rate of $2 \mathrm{kHz}$. Pull-off distances of more than $40 \mathrm{~nm}$ were used in all cases to minimize tip-solid substrate interactions. An on-surface trigger force of about $2-3 \mathrm{nN}$ was used. An approach and retraction velocity of $200 \mathrm{~nm} \mathrm{~s}^{-1}$ was used in all cases except for the experiments concerning the rate dependency of the mechano-isomerization in P1. In these experiments retraction velocities of up to $2 \mu \mathrm{m} \mathrm{s}^{-1}$ were used for which the sampling rate was increased to $10 \mathrm{kHz}$. Experiments with P1 were performed on EF-silica, gold and MF-gold using EF-tips and gold tips. Experiments with $\mathbf{P 2}$ were performed on EF-silica using EF-tips. Experiments with the rest of the polymers were performed on silica using silicon tips. All experiments were carried out at a temperature of $25{ }^{\circ} \mathrm{C}$.

The force versus extension profiles of the polymers were obtained by pulling experiments using the AFM. A normalization procedure was used to ascertain that the force versus extension profiles were from single molecules. This procedure consists of dividing the extension of the polymer, $x$, by its extension at force $F_{0}$, i.e. $x_{0}\left(F_{0}\right)$. The extension $x_{0}$ is proportional to the contour length of the polymer. Similarly, $x_{0}$ varies from one pulling event to the other, because the polymers are normally attached to the AFM tip randomly along the polymer backbone. When the extension of the polymer $x$ is normalized by $x_{0}$, the normalized extension $x / x_{0}$ becomes independent of the contour length. The resulting normalized force versus extension profile, in particular, force $F$ as a function of the normalized extension $x / x_{0}$, can be used to identify single molecule extensions. When the normalized force versus extension profiles overlap, single molecule extension responses are assumed.

We used the modified freely jointed chain (FJC) model to fit the force versus extension profiles. The FJC model, in addition to the linear elastic regime at low forces, describes the nonlinear regime at high forces. The extension $x$ is expressed in terms of the force $F$ as: ${ }^{20}$

$$
x=L\left[\operatorname{coth}\left(\frac{\ell F}{k_{\mathrm{B}} T}\right)-\frac{k_{\mathrm{B}} T}{\ell F}+\frac{F}{K}\right]
$$

where $L$ is the contour length, $k_{\mathrm{B}}$ the Boltzmann constant, $T$ the absolute temperature, $\ell$ the Kuhn length, and $K$ the elasticity constant. In general, the elastic response of polymer molecules enters the nonlinear FJC deformation regime at high forces. ${ }^{21}$ The FJC is also commonly used in the modelling of the force response of synthetic polymers. ${ }^{22}$

AFM imaging of P1 was performed using a Cypher AFM in amplitude modulation mode. Silicon tips with a nominal tip radius $<10 \mathrm{~nm}$ and resonance frequencies around $25 \mathrm{kHz}$ in DMSO and $130 \mathrm{kHz}$ in air were used. The cantilever was externally driven by an AC modulated blue laser close to its resonance frequency in DMSO. To create a dilute polymer film on mica (Plano, Germany), P1 was adsorbed from a solution of $1 \mathrm{mg} \mathrm{L}{ }^{-1}$ concentration in DMSO for $40 \mathrm{~s}$ after which the polymer solution was replaced with a larger volume of the solvent. A scan rate of $7.8 \mathrm{~Hz}$ with a free oscillation amplitude (FOA) of about $10 \mathrm{~nm}$ and an amplitude set-point of about 76\% of FOA was used to obtain the AFM image.

\section{Results and discussion}

We examined the effect of mechanical force on cis-to-trans isomerization of the carbon-carbon double bond at the level of single polymer molecules using AFM. Three polymers with cis double bonds in the backbone, namely $\mathbf{P 1}$, cis-PB and $\mathbf{P B}$, were investigated. As control, we examined three polymers without double bonds in the backbone, namely $\mathbf{P 2}, \mathbf{P E}$ and PS. The structures of these polymers are shown in Fig. 1.

\section{Detecting cis-to-trans isomerization events}

Using AFM we pulled single molecules of $\mathbf{P 1}$ and obtained their force versus extension profiles. In the majority of cases, the molecules detached from the AFM tip at forces below a few hundred pN. If a polymer remained attached at higher forces around $1 \mathrm{nN}$, in about $50 \%$ of the occurrences, it underwent a sudden transition as revealed by an extension increase in the force versus extension profile. An example is provided in Fig. 2. The extension increase results in a drop in the probed force, where the AFM cantilever snaps to a new position that is more separated from the surface. At extensions below this transition, the restoring force due to $\mathbf{P 1}$ backbone rigidity increases with the extension of the polymer. When the restoring force is high enough, multiple cis double bonds isomerize to trans double bonds. Since the trans isomer is longer than the cis isomer by 


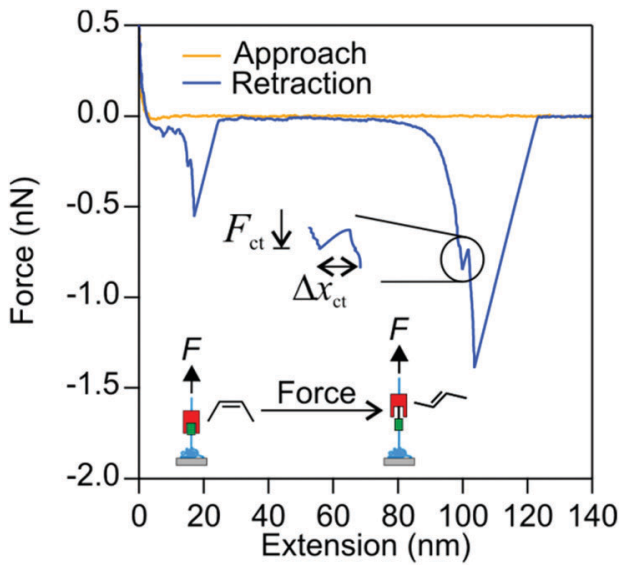

Fig. 2 P1 isomerizes under mechanical force. The onset of isomerization is denoted by the extension increase $\Delta x_{\mathrm{ct}}$ and by the force $F_{\mathrm{ct}}$. The polymer elongates after isomerization since trans isomers are longer than cis isomers. The added length to the polymer results in relaxation in the probed force. A mechanochemistry view of the isomerization process in the carbon-carbon double bond is illustrated in the figure.

about $120 \mathrm{pm}$, the isomerization results in an increase in the extension of the polymer. The extension increase is denoted by $\Delta x_{\mathrm{ct}}$. The force where the extension increase occurs is denoted by the isomerization force $F_{\text {ct }}$. After the isomerization, the polymer is further extended before it isomerizes again or detaches from the AFM tip.

It is important to ensure that the observed transition originates from a single molecule that is firmly anchored between the AFM tip and the solid substrate. We do this by first obtaining the elastic response of a single molecule of $\mathbf{P 1}$. Thereby we initially collect all force versus extension profiles of the polymer in which no isomerization was observed. A few examples of force versus extension profiles with no isomerization are shown in Fig. 3(a). These experiments were repeated on EF-silica, gold and MF-gold and the resulting force versus extension profiles were normalized at $300 \mathrm{pN}$ and compared in Fig. 3(b). Each force versus extension profile was fitted to the FJC model (eqn (1)). The fitted parameters are Kuhn length $\ell=0.53 \pm 0.06 \mathrm{~nm}$ and elasticity constant $K=20 \pm 3 \mathrm{nN}$. In Fig. 3(b) an FJC curve that is reproduced using the mean values of the Kuhn length and the elasticity constant and normalized at $300 \mathrm{pN}$ is also shown. The compatibility of different force versus extension profiles of $\mathbf{P 1}$ is calculated by means of the difference between each profile and the FJC curve. The good agreement between the force versus extension profiles guarantees that a single polymer molecule was pulled in each case. As a quantitative indicator of the goodness of the comparisons between the force versus extension profiles and the FJC curve, the reduced $\tilde{\chi}^{2}$ statistics was used. This quantity is defined as:

$$
\tilde{\chi}^{2}=\sum_{i}\left(F_{\mathrm{EXP}}^{(i)}-F_{\mathrm{FJC}}^{(i)}\right)^{2} /\left(N \sigma^{2}\right)
$$

where $F_{\text {EXP }}^{(i)}$ is the experimental force, and $F_{\text {FJC }}^{(i)}$ the FJC force that was calculated using the mean values of the Kuhn length and the elasticity constant. The index $i=1, \ldots, N$ labels the (a)



(b)
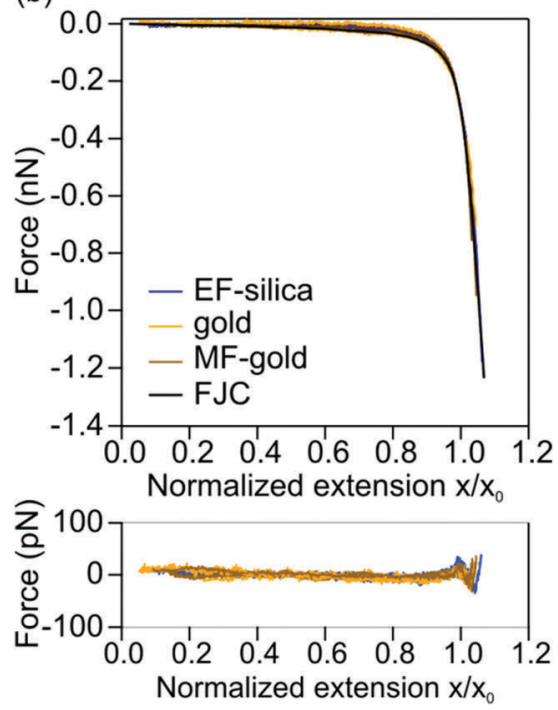

Fig. 3 (a) Force versus extension profiles of $\mathbf{P} \mathbf{1}$ with no isomerization. The no-isomerization profiles are used to obtain the elastic response of a single molecule of $\mathbf{P} \mathbf{1}$. Orange is a typical approach and 1 to 4 are different retraction curves. (b) Normalized force versus extension profiles of $\mathbf{P} \mathbf{1}$ on various solid substrates together with the FJC curve that is reproduced using the mean values of the Kuhn length and the elasticity constant, $\ell=0.53 \mathrm{~nm}$ and $K=20 \mathrm{nN}$, respectively. Normalization of the profiles and the FJC curve is at $300 \mathrm{pN}$. The difference between each profile and the FJC curve is shown below. The reduced $\tilde{\chi}^{2}$ varies from 1 to 12 .

data points, where $N$ is the total number of data points. The standard deviation $\sigma=4 \mathrm{pN}$ is smaller than the one calculated from the equipartition theorem, which is around $20 \mathrm{pN}$. This reduction is due to the application of a low-pass filter at $2 \mathrm{kHz}$ to the deflection data.

In every force versus extension profile in which a transition was observed, we compared the pre-transition section of the force versus extension profile with the elastic response of a single polymer molecule. For practical purposes, the FJC curve is reproduced using the mean values of the Kuhn length, $\ell=0.53 \mathrm{~nm}$, and the elasticity constant, $K=20 \mathrm{nN}$, of $\mathbf{P 1}$ is then used to compare with the pre-transition sections. In the case of disagreement, the force versus extension profile was discarded. The reader is reminded that the application of the FJC to evaluate force versus extension profiles with transitions is for illustration purposes only. The pre-transition sections can be directly compared with the elastic response of a single molecule of P1 as obtained in Fig. 3. Fig. 4(a) shows the 
normalized pre-isomerization sections of different force versus extension profiles of P1, which compare well with each other and with the FJC curve. The good agreement obtained ensures that the analyzed profiles are from single polymer molecules. The post-isomerization sections of the profiles are shown in Fig. 4(b).

To ensure that the observed transitions were not due to specific polymer-surface interactions, such as formation and breaking of multiple adhesion sites, we measured the isomerization force $F_{\mathrm{ct}}$ with different tip and solid substrate chemistries. Thereby, the EF-tip was used against EF-silica, gold and MF-gold, and the gold tip was used against EF-silica. We expect that specific polymer-surface interactions would indicate a dependence of the observed transition forces on the chemical signature of the AFM tip and the solid substrate. Fig. 5(a) shows that $F_{\text {ct }}$ values are the same within standard deviation for different surface

(a)

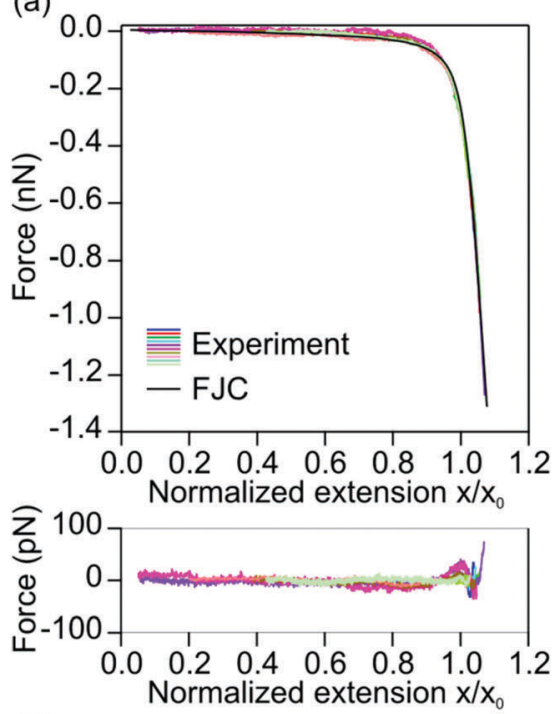

(b)

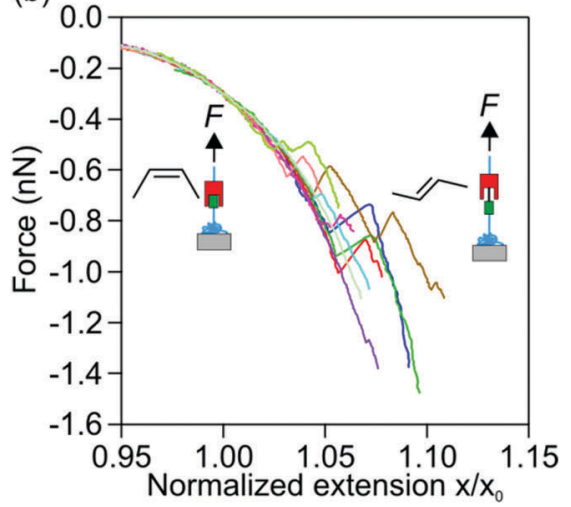

Fig. 4 Normalized force versus extension profiles of $\mathbf{P} \mathbf{1}$ upon isomerization. (a) Pre-isomerization sections together with the FJC curve. The FJC curve is reproduced using mean values of the Kuhn length and the elasticity constant of $\mathbf{P} \mathbf{1}, \ell=0.53 \mathrm{~nm}$ and $K=20 \mathrm{nN}$, respectively. Normalization of the profiles is at $300 \mathrm{pN}$. The difference between each profile and the FJC curve is shown below. The reduced $\tilde{\chi}^{2}$ varies from 1.3 to 8.6. (b) Post-isomerization sections of the profiles shown in (a). A scheme of the cis-to-trans isomerization event is also shown.

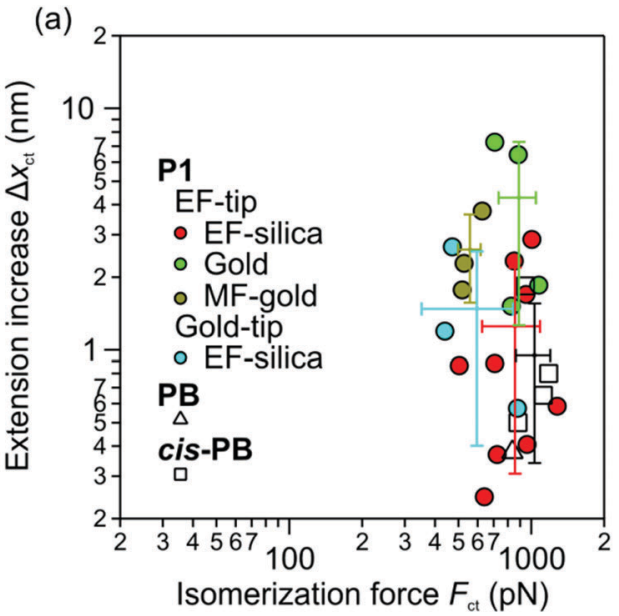

(b)



Isomerization force $F_{\mathrm{ct}}(\mathrm{pN})$ (c)

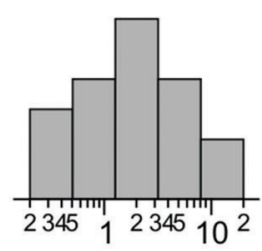

Extension increase $\Delta x_{\mathrm{ct}}(\mathrm{nm})$
Fig. 5 (a) The extension increase $\Delta x_{c t}$ of P1, cis-PB, and PB plotted versus the isomerization force $F_{\mathrm{ct}}$. The error bars associated with isomerization of P1 on EF-silica, gold and MF-gold using EF-tips, on EF-silica using gold tips and isomerization of cis-PB represent standard deviations in the measured isomerization forces and extension increase in each dataset. $F_{\mathrm{ct}}$ agrees reasonably for tips and solid surfaces that are chemically different. Histogram of isomerization forces (b) and extension increase (c) measured for P1.

chemistries of the solid substrates and the AFM tips. This observation further ascertains that the observed transitions are due to intramolecular conversion of cis double bonds to trans double bonds. Our data give a force of $800 \pm 60 \mathrm{pN}$ for the isomerization of the double bond. This force is lower than the force associated with breaking of covalent bonds and rings (1-2 $\mathrm{nN})$ that have been obtained experimentally. ${ }^{9-11,23-25}$ Additional results are provided in the ESI $\dagger$ (Tables S1, S2 and Fig. S5, S6).

Force response of other polymers with cis double bonds in the backbone

Cis-to-trans isomerization of cis-PB and PB was also investigated. The force versus extension profiles of cis-PB and $\mathbf{P B}$ exhibited isomerization transitions akin to the response of P1. Similar to analysis for $\mathbf{P 1}$, we used the no-isomerization force versus extension profiles of each polymer to obtain the elastic response of its single molecules. Fitting to the FJC model resulted in the Kuhn lengths $\ell=0.33 \pm 0.03 \mathrm{~nm}$ and $0.44 \pm 0.02 \mathrm{~nm}$, and the elasticity constants $K=25 \pm 4 \mathrm{nN}$ and $16 \pm 2 \mathrm{nN}$, for cis-PB and PB, respectively. The slightly higher Kuhn length in the case of PB might be due to the higher percentage of trans double bonds in the PB backbone. Comparisons between the pre-isomerization sections of the force versus extension profiles and responses of single molecules showed agreement in each case. We find that 
these polymers have cis-to-trans isomerization forces $F_{\text {ct }}$ in the same range as measured for P1. This is shown in Fig. 5(a). Experimental results for cis-PB and PB can be found in the ESI $\dagger$ (Fig. S7-S9).

\section{Force response of polymers without double bonds in the backbone}

We extended our investigations to polymers that were structurally similar to P1, PB and cis-PB but contained no double bonds in the backbone. Among them $\mathbf{P 2}$ is structurally similar to P1, and PE is structurally similar to PB and cis-PB. We found that the force versus extension profiles of P2, PS and PE did not show any evidence of a transition similar to that observed for P1, PB and cis-PB. In fact, if a transition occurred, it was possible to show that the transition was not due to a single molecule. This finding justifies our attribution of the observed transitions in the force versus extension profiles of $\mathbf{P 1}, \mathbf{P B}$ and cis-PB to the isomerization of cis double bonds. For P2 a Kuhn length $\ell=0.48 \pm 0.04 \mathrm{~nm}$ and a elasticity constant $K=10 \pm 1 \mathrm{nN}$ were found by fitting to the FJC model while these values were $\ell=0.56 \pm 0.04 \mathrm{~nm}$ and $K=30 \pm 5 \mathrm{nN}$ for PE, and $\ell=0.81 \pm 0.06 \mathrm{~nm}$ and $K=47 \pm 5 \mathrm{nN}$ for PS. Among these polymers the elastic properties of PS have been studied previously. In toluene, a Kuhn length of $1.2 \mathrm{~nm}$ and a elasticity constant of $2.6 \mathrm{nN}$ were found, ${ }^{26}$ while in isopropanol, they were found to be $0.4 \mathrm{~nm}$ and $7.2 \mathrm{nN}$, respectively. The difference between the elastic properties is potentially due to the different chemical properties of the solvents. The higher Kuhn length of PS compared to PE is due to the phenyl side groups in PS that hinder rotation of the chain by steric repulsion which is an entropic effect. The higher elasticity constant in PS compared to PE hints that the phenyl groups hinder bending of the bond angles. More interestingly we find that the double bonds in PB and cis-PB do not increase the elasticity constant of these chains. This might be due to the bending of bond angles being the main source of elasticity in these chains as compared with stretching of bonds. The higher percentage of hydrogen in $\mathbf{P E}$ then results in the higher elasticity constant of $\mathbf{P E}$ compared to $\mathbf{P B}$ and cis-PB. We also find that the elasticity constant of $\mathbf{P 2}$ is lower than that of the other polymers. While the lower Kuhn length of $\mathbf{P 2}$ compared to $\mathbf{P E}$ and $\mathbf{P S}$ can be a solvent effect, we attribute the lower elasticity constant to the lower range of the probed forces in $\mathbf{P 2}(<500 \mathrm{pN})$. The force versus extension profiles of P2, PS and PE are shown in the ESI $\dagger$ (Fig. S10-S13).

\section{Interpreting mechanically induced cis-to-trans isomerization}

Fig. 2 and 4(b) show sudden relaxation in force upon isomerization of cis double bonds. This characteristic response, among others, resembles the mechano-isomerization of prolyl. ${ }^{12,13}$ Observation of a sudden relaxation ${ }^{10,15-19}$ or a plateau ${ }^{9,14}$ in the force versus extension profiles of force-sensitive polymers depends mainly on the shape of the energy landscape and the kinetics of transition along the pulling coordinate of the mechanophore, the number of transitions, and the mechanical response of the pulling probe. ${ }^{27,28}$ For P1, PB and cis-PB the polymer chain relaxes since its length increases as multiple cis double bonds isomerize to trans double bonds. This process is much faster than the response time of the AFM cantilever, $\sim 20 \mu \mathrm{s}$, and thus the cantilever snaps to a new position.

It is possible to estimate an upper limit of the force for the isomerization of the carbon-carbon double bond: when force is aligned with the reaction coordinate, the activation energy $E_{\mathrm{b}}$ is lowered by an amount $F \Delta x^{*}$, where $\Delta x^{*}$ is the transition length. Without thermal activation, the isomerization becomes spontaneous at force $F=E_{\mathrm{b}} / \Delta x^{*}$. The experimentally measured activation energy for cis-to-trans isomerization of the carboncarbon double bond for various organic molecules in liquid and gas is $E_{\mathrm{b}}=100-260 \mathrm{~kJ} \mathrm{~mol}^{-1} \cdot{ }^{4,29}$ The lower limit of the activation energy is attributed to spin-forbidden crossing from the singlet to triplet state. A value of about $170 \mathrm{~kJ} \mathrm{~mol}^{-1}$ is generally attributed to adiabatic rotational transition, ${ }^{30}$ and shall be used in the following. A transition length $\Delta x^{*}=50 \mathrm{pm}$ can be estimated from the difference in the carbon-carbon distance between the trans isomer, $L_{\text {trans }}=0.4 \mathrm{~nm}$, and the cis isomer, $L_{\text {trans }}=0.3 \mathrm{~nm}$, of 2-butene assuming a symmetric reaction coordinate. Using these values, one obtains a force of about $5 \mathrm{nN}$. However, this value is an overestimation as it approaches the rupture forces of the silicon-oxygen covalent bond, $5.2 \mathrm{nN}$, the silicon-carbon covalent bond, $4.8 \mathrm{nN}$, and the silicon-nitrogen covalent bond, $4.7 \mathrm{nN}$, and even exceeds the rupture force of the silicon-silicon covalent bond, $3.3 \mathrm{nN} .{ }^{23} \mathrm{We}$ will nevertheless use this estimation to compare with the experimentally obtained isomerization force. This estimation is about 6-times larger than we measured experimentally.

As the activation energy diminishes along the reaction coordinate, the lifetime of the bond decreases by a respective Boltzmann factor. In terms of reciprocal of the lifetime: ${ }^{31}$

$$
k(F)=k_{0} \exp \left(\frac{F \Delta x^{*}}{k_{\mathrm{B}} T}\right)
$$

where $k$ is the force-dependent rate and $k_{0}$ the thermal rate.

One possibility for the difference between the measured isomerization force and the approximation above can be the norbornene in the backbone of P1. Norbornene increases the transition length $\Delta x^{*}$ to $60.5 \mathrm{pm} .{ }^{11}$ This is calculated from the difference in the contour lengths of the trans isomer, $L_{\text {trans }}=$ $1.24 \mathrm{~nm}$, and the cis isomer, $L_{\text {trans }}=1.12 \mathrm{~nm}$, of a monomer of P1 assuming a symmetric reaction coordinate. Thereby the rate of isomerization is increased 10 -fold at the isomerization force of $800 \mathrm{pN}$. The effect of longer transition lengths on reducing reaction forces has been previously shown in the measurements of opening of covalent rings, ${ }^{11}$ and is known as the molecular lever-arm effect. Asymmetry of reaction coordinates has a similar effect. More specifically the transition length can be longer than the simple estimations above for symmetric reaction coordinates, or even change with force. ${ }^{32}$

More importantly, while the mechanical force reduces the activation energy, the barrier might not completely disappear. In this case, isomerization could only occur by thermal activation over an existing barrier. This effect is similar to the previous measurements on breaking of covalent bonds and rings in solvents and at room temperature where deviations between experimental and estimated rupture forces have also been found. ${ }^{9-11,23-25}$ 
For example the silicon-carbon bond has a rupture force of $2.0 \mathrm{nN}$ experimentally, while a rupture force of about $4.8 \mathrm{nN}$ was obtained for this bond by neglecting thermal activation using density functional theory (DFT). ${ }^{33}$ Intramolecular interactions in the highly stretched polymer molecule as well as effects from solvent molecules can contribute to the lower isomerization force.

Fig. 5 illustrates the distribution of the isomerization forces. The reason that such a distribution is being observed is that the timescale of the isomerization is sufficiently slower than the one of the AFM experiment. The present mechano-isomerization experiments are at rates that are much faster than the thermal rate of isomerization of the carbon-carbon double bond, which is generally below $10^{-8} \mathrm{~s}^{-1} \cdot{ }^{29}$ In our experiments, the time frame of AFM pulling varied between 0.1 and $2 \mathrm{~s}$, during which the polymer was under an increasing mechanical force prior to isomerization. The variable experimental time frame depends, in addition to the force, on the contour length of the polymer, the pulling velocity, and the stiffness of the cantilever and the polymer. Using the response time of the AFM cantilever, $\sim 20 \mu \mathrm{s}$, as the slowest commendable timescale for the isomerization in our experiments, it is evident that these experiments are extremely out of equilibrium. Using DFT, it has been shown that rupture forces of covalent bonds depend on the lifetime of the bond which also varies with the force. ${ }^{33}$ Thereby a distribution of rupture forces was found for 7 different covalent bonds at a constant force rate of $10 \mathrm{nN} \mathrm{s}^{-1} \cdot 33$ The distribution of isomerization forces is similar to the distributions of rupture forces in the covalent bonds. ${ }^{23}$ The non-equilibrium nature of these experiments, the variable time frame and thermal fluctuations explain the deviations from the mean isomerization force measured for $\mathbf{P 1} .^{34}$

The peak of the distribution or the most probable force $F^{*}$ is described by the Bell-Evans model: ${ }^{35}$

$$
F^{*}=\frac{k_{\mathrm{B}} T}{\Delta x^{*}} \ln \left(\frac{\Delta x^{*} \mathrm{~d} F / \mathrm{d} t}{k_{0} k_{\mathrm{B}} T}\right)
$$

where $\mathrm{d} F / \mathrm{d} t$ is the force rate. Following this model, we also tried to vary the force rate by performing experiments at different pulling velocities ranging from 50 to $2000 \mathrm{~nm} \mathrm{~s}^{-1}$. Velocities lower than $50 \mathrm{~nm} \mathrm{~s}^{-1}$ would not result in stable force versus extension profiles due to the inherent thermal drifts in the AFM apparatus. In this range of velocities we did not observe a variation in the elastic response of $\mathbf{P 1}$. This finding shows, on the one hand, that there is no significant change in the lubrication forces on the cantilever as a function of the pulling velocity. The velocity-independent elastic response of $\mathbf{P 1}$ also suggests that the extension of the polymer is under nearequilibrium conditions with no significant intrachain friction within the range of the explored velocities. The viscous component of the mechanical response of single molecules has been previously measured. ${ }^{36}$ In this work however we only require the elastic component.

Here again in the majority of force versus extension profiles the molecule detached at forces only as high as a few hundred pN. For the remaining chains, we find that the isomerization force remains constant over the force-rate range from 4 to $12 \mathrm{nN} \mathrm{s}^{-1}$.
This behaviour is expected since there is a logarithmic dependence of the most probable force on the force rate (eqn (4)). Thus one needs to explore the transition forces over orders of magnitude in the force rate to observe an effect. While this procedure has been actively used for weak bonds, ${ }^{17,37-40}$ for strong bonds with reaction forces close to the $\mathrm{nN}$ range, $, 9,10,23-25$ the application of the model is experimentally not feasible. The strongest tethers in AFM single molecule force spectroscopy are covalent bonds which have a strength of only a few $n .^{23,24}$ We however speculate that if we were able to change the force rate over orders of magnitude, we would observe the force-rate dependency of the most probable isomerization force. For chemical transitions that are nonequilibrium, the most probable force is expected to increase with the force rate. ${ }^{35}$

Fig. 5(a) shows that extension increase $\Delta x_{\text {ct }}$ varies from 0.3 to $7.3 \mathrm{~nm}$. The smallest possible extension increase from one cis double bond to one trans double bond in P1 is about $0.12 \mathrm{~nm}$. A larger extension increase observed in the experiments is due to isomerization of multiple cis double bonds. We suspect that sections of several neighbouring cis and trans double bonds coexist in the P1 structure. The larger extension increase corresponds to sections with several neighbouring cis double bonds or when multiple sections of all cis double bonds isomerize simultaneously. Variations in the contour length of the pulled polymers also result in variations in the extension increase since the number of available cis monomers varies with the contour length. These effects result in a larger variation in the extension increase (Fig. 5(c)) as compared with the isomerization force (Fig. 5(b)). The isomerization force is found to have no detectable correlation with the number of isomerized cis monomers (Fig. 5(a)). The extension increase, or the number of isomerized cis monomers, can be normalized by the total number of available cis monomers within the contour length of the pulled molecule. We found that in almost all cases, a complete isomerization of cis monomers did not occur. However P1 generally had a higher percentage of isomerization as compared with $\mathbf{P B}$ and cis-PB.

It was mentioned earlier that the characteristic response of the isomerization of cis double bonds in our measurements was a sudden force relaxation. This response is akin to the response of opening of modular domains in proteins such as titin and tenascin. ${ }^{15,16}$ We thereby performed AFM imaging of P1 to gain an understanding of its conformations in DMSO. Fig. 6 shows that $\mathbf{P 1}$ has random coil conformation. No high-order structure is also visible in the AFM image. The phenomenon of force-induced opening of high order structures does not correspond to P1.

The polymers without double bonds in the backbone were chosen according to the criterion of having similar structures to the polymers with cis double bonds in the backbone. It is possible that the polymers with cis double bonds in the backbone form aggregates or clusters on the substrate. In this case the observed transitions could be due to the breakage of physical bonds between different polymer chains. In this case, we expect to observe more frequent transitions in the force versus extension profiles. This is because in the case of aggregate formation, the polymer chain forms many adhesion points with the neighbouring chains. In contrast we most often observed a 


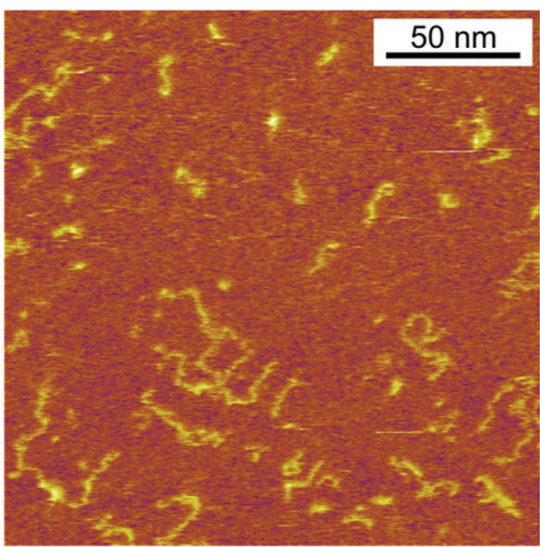

Fig. 6 AFM image of P1 shows random coil conformation and no highorder structure.

single transition in the force versus extension profiles. In addition, since the polymers without double bonds in the backbone were structurally similar to the polymers with cis double bonds in the backbone, they were prone to form aggregates or clusters on the substrate. Nevertheless, the lack of observation of similar transitions in polymers without double bonds in the backbone as was observed for the polymers with cis double bonds in the backbone reassures that the observed transitions are not due to breakage of aggregates.

Let us stress the various interesting aspects of P1 over PB and cis-PB. The monomers in P1 can be functionalized with chemically reactive subunits due to the high functional group tolerance of the Grubbs 3rd generation ruthenium complex used. We found that the probability of the mechanically induced isomerization varies among these polymers. These probabilities were $17 \%$ for PB and 17\% for cis-PB, and 53\% for P1. We also found that the extension increase $\Delta x_{\text {ct }}$ and the percent isomerization were generally higher in P1 than cis-PB and PB. These observations suggest that the molecular structure of $\mathbf{P 1}$ is more desirable for mechano-isomerization of double bonds. Further measurements and data analysis in support of our discussions can be found in the ESI† (Fig. S14-S16).

\section{Conclusions}

Cis-to-trans isomerization of carbon-carbon can be induced by the application of mechanical force. We show this by using AFM and at the level of single polymer molecules which contained cis double bonds in the backbone. We found that the isomerization force is about $800 \mathrm{pN}$. Our results open the possibility to develop a new class of force-responsive materials based on mechano-isomerization of carbon-carbon double bonds.

\section{Acknowledgements}

The authors gratefully acknowledge a useful discussion with Thorsten Hugel. Support of this research was provided by the National Competence Center in Research (NCCR) for Bio-Inspired Materials, and University of Geneva.

\section{Notes and references}

1 C. Dugave and L. Demange, Chem. Rev., 2003, 103, 2475-2532.

2 K. Palczewski, J. Biol. Chem., 2012, 287, 1612-1619.

3 R. E. Dawson, S. F. Lincoln and C. J. Easton, Chem. Commun., 2008, 3980-3982.

4 D. Masson, C. Richard and R. Martin, Int. J. Chem. Kinet., 1976, 8, 37-44.

5 S. K. Sharma, V. K. Srivastava and R. V. Jasra, J. Mol. Catal. A: Chem., 2006, 245, 200-209.

6 R. A. Johnson and S. Seltzer, J. Am. Chem. Soc., 1973, 95, 5700-5706.

7 Q. Sun, C. Zhang, L. Wang, Z. Li, A. Hu, Q. Tan and W. Xu, Chem. Commun., 2014, 50, 1728-1730.

8 Y. Liu, K. Liu, Z. Wang and X. Zhang, Chem. - Eur. J., 2011, 17, 9930-9935.

9 J. Wang, T. B. Kouznetsova, Z. Niu, M. T. Ong, H. Klukovich, A. L. Rheingold, T. J. Martinez and S. L. Craig, Nat. Chem., 2015, 7, 323-327.

10 D. Schuetze, K. Holz, J. Mueller, M. K. Beyer, U. Luening and B. Hartke, Angew. Chem., Int. Ed., 2015, 54, 2556-2559.

11 H. M. Klukovich, T. B. Kouznetsova, Z. S. Kean, J. M. Lenhardt and S. L. Craig, Nat. Chem., 2013, 5, 110-114.

12 A. Valiaev, D. W. Lim, T. G. Oas, A. Chilkoti and S. Zauscher, J. Am. Chem. Soc., 2007, 129, 6491-6497.

13 L. Rognoni, T. Most, G. Zoldak and M. Rief, Proc. Natl. Acad. Sci. U. S. A., 2014, 111, 5568-5573.

14 P. E. Marszalek, A. F. Oberhauser, Y. P. Pang and J. M. Fernandez, Nature, 1998, 396, 661-664.

15 M. Rief, M. Gautel, F. Oesterhelt, J. M. Fernandez and H. E. Gaub, Science, 1997, 276, 1109-1112.

16 A. F. Oberhauser, P. E. Marszalek, H. P. Erickson and J. M. Fernandez, Nature, 1998, 393, 181-185.

17 N. Hosono, A. M. Kushner, J. Chung, A. R. A. Palmans, Z. Guan and E. W. Meijer, J. Am. Chem. Soc., 2015, 137, 6880-6888.

18 J. Liphardt, S. Dumont, S. B. Smith, I. Tinoco and C. Bustamante, Science, 2002, 296, 1832-1835.

19 A. N. Gupta, A. Vincent, K. Neupane, H. Yu, F. Wang and M. T. Woodside, Nat. Phys., 2011, 7, 631-634.

20 A. Janshoff, M. Neitzert, Y. Oberdorfer and H. Fuchs, Angew. Chem., Int. Ed., 2000, 39, 3213-3237.

21 A. V. Dobrynin, J.-M. Y. Carrillo and M. Rubinstein, Macromolecules, 2010, 43, 9181-9190.

22 M. I. Giannotti and G. J. Vancso, ChemPhysChem, 2007, 8, 2290-2307.

23 M. Grandbois, M. Beyer, M. Rief, H. Clausen-Schaumann and H. E. Gaub, Science, 1999, 283, 1727-1730.

24 L. Garnier, B. Gauthier-Manuel, E. W. van der Vegte, J. Snijders and G. Hadziioannou, J. Chem. Phys., 2000, 113, 2497-2503.

25 D. Wu, J. M. Lenhardt, A. L. Black, B. B. Akhremitchev and S. L. Craig, J. Am. Chem. Soc., 2010, 132, 15936-15938.

26 H. Kikuchi, N. Yokoyama and T. Kajiyama, Chem. Lett., 1997, 1107-1108. 
27 G. Hummer and A. Szabo, Biophys. J., 2003, 85, 5-15.

28 M. Rief, J. M. Fernandez and H. E. Gaub, Phys. Rev. Lett., 1998, 81, 4764-4767.

29 J. L. Magee, W. Shand and H. Eyring, J. Am. Chem. Soc., 1941, 63, 677-688.

30 P. Bortolus and G. Cauzzo, Trans. Faraday Soc., 1970, 66, 1161-1164.

31 G. I. Bell, Science, 1978, 200, 618-627.

32 D. E. Makarov, J. Chem. Phys., 2016, 144, 030901.

33 M. K. Beyer, J. Chem. Phys., 2000, 112, 7307-7312.

34 C. Bustamante, J. Liphardt and F. Ritort, Phys. Today, 2005, 58, 43-48.
35 E. Evans and K. Ritchie, Biophys. J., 1997, 72, 1541-1555.

36 M. Radiom, M. R. Paul and W. A. Ducker, Nanotechnology, 2016, 27, 255701.

37 F. Kienberger, G. Kada, H. J. Gruber, V. P. Pastushenko, C. Riener, M. Trieb, H.-G. Knaus, H. Schindler and P. Hinterdorfer, Single Mol., 2000, 1, 59-65.

38 A. Embrechts, A. H. Velders, H. Schoenherr and G. J. Vancso, Langmuir, 2011, 27, 14272-14278.

39 F. R. Kersey, W. C. Yount and S. L. Craig, J. Am. Chem. Soc., 2006, 128, 3886-3887.

40 M. Gensler, C. Eidamshaus, M. Taszarek, H.-U. Reissig and J. P. Rabe, Beilstein J. Org. Chem., 2015, 11, 817-827. 